\section{Frederico Simões Barbosa: uma trajetória de contribuições à ciência e à saúde pública}

\author{
Frederico Simões Barbosa: a history of \\ contributions to science and public health \\ Frederico Simões Barbosa: un historial de
contribuciones a la ciencia y la salud pública
}

${ }^{1}$ Casa de Oswaldo Cruz, Fundação Oswaldo Cruz, Rio de Janeiro, Brasil.

Correspondência

P. R. E. Santos

Casa de Oswaldo Cruz,

Fundação Oswaldo Cruz.

Av. Brasil 4365, Rio de

Janeiro, RJ 21040-900, Brasil.

elian@coc.fiocruz.br
Como definir Frederico Simões Barbosa, que na visão de Carlos Coimbra Jr. 1, foi uma das figuras mais notáveis da epidemiologia e da saúde pública no país em meados do século XX? Ao longo de mais de cinco décadas, como pesquisador, professor e gestor de instituições científicas e universidades, Frederico Simões Barbosa conheceu os mais diversos espaços institucionais. Percorreu os caminhos das salas de aulas, laboratórios, encontros científicos, gabinetes, regiões rurais e urbanas do país, conviveu com inúmeros outros personagens e foi um ator inquieto e empreendedor no processo de institucionalização da saúde pública como campo de conhecimento no país. Nosso personagem nasceu na cidade do Recife (Pernambuco) em 27 de julho de 1916, filho de Fernando Simões Barbosa e Maria Schmidt Simões Barbosa. Seguindo a tradição de seu pai e de seu avô, ambos médicos, formou-se em 1938 pela Faculdade de Medicina do Recife (FMR), cuja geração teve como forte influência as mudanças deflagradas após as conquistas de Oswaldo Cruz e do Instituto de Manguinhos nos campos da medicina experimental e das práticas sanitárias. Antes mesmo de concluir o curso de medicina conheceu o sanitarista Samuel Pessoa (sobre a trajetória do médico parasitologista Samuel Pessoa, ver Paiva 2), que em 1936 esteve no Recife para ministrar um curso de parasitologia médica e teve influência decisiva na sua opção profissional. Em entrevista, revelou sua admiração por traços do professor da Universidade
Paulo Roberto Elian dos Santos 1 Francisco dos Santos Lourenço 1 Nathacha Regazzini Bianchi Reis ${ }^{1}$

de São Paulo (USP), que definiriam sua filiação a uma "Escola" de formação de parasitologistas.

"O curso foi excelente e, pela primeira vez, tive um contato íntimo com o professor Samuel Pessoa. Sua visita foi muito importante e logo começamos a trabalhar. Fundamos então a Sociedade Pernambucana de Biologia (...) e formamos um pequeno grupo para trabalhar em parasitologia. (...) Samuel era uma pessoa extremamente extrovertida, tinha um carisma fantástico e conduzia o trabalho com muita liberdade, dentro do laboratório. Era um scholar de fato. Ele foi o formador de uma Escola, uma figura realmente extraordinária" 1 (p. 147).

Concluído o curso de medicina, Frederico Simões Barbosa integrou um grupo de jovens estudantes pernambucanos que obteve bolsas de estudos para se especializarem nos diferentes campos das ciências biomédicas, e assim, a partir de 1939, passou dois anos na USP estudando parasitologia e micologia sob a orientação de Samuel Pessoa e Floriano de Almeida, respectivamente 1 .

Ao retornar a Recife vinculou-se à FMR. Engajou-se tanto na vida acadêmica, na qual foi responsável pelas cadeiras de microbiologia, parasitologia, zoologia e medicina preventiva, quanto no desenvolvimento de pesquisas e políticas públicas voltadas para as condições de saúde de sua região ao longo das décadas de 1940 e 1950. Essa trajetória denota o interesse de Frederico Simões Barbosa pelas ciências biológicas, que o 


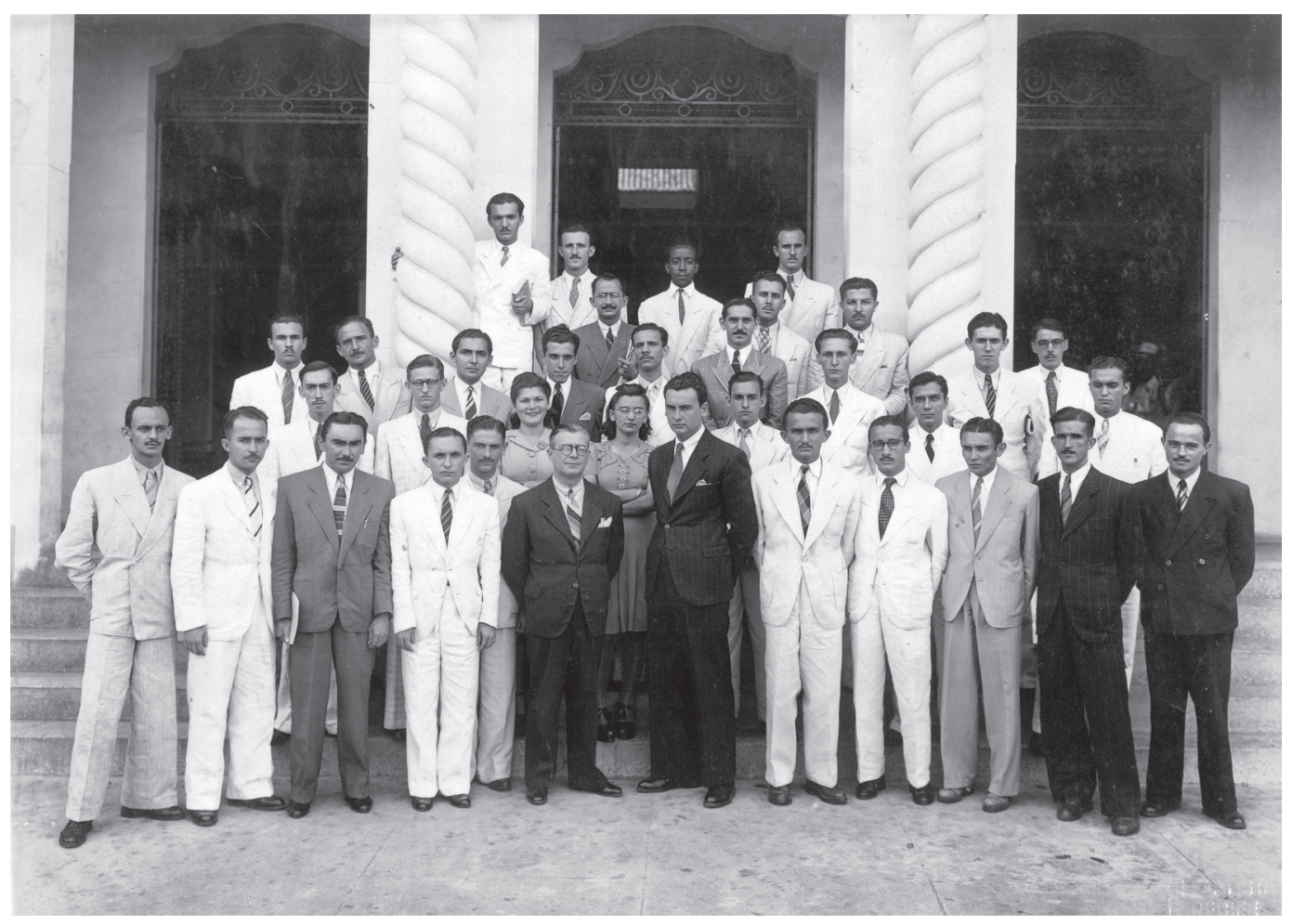

fez declinar da clínica médica, seguindo outros caminhos mais condizentes com a sua vocação de pesquisador e professor. Em 1952, realizando vontade adiada por razões familiares e circunstanciais, graduou-se em história natural na Faculdade Católica de Pernambuco.

O foco de suas investigações foi a esquistossomose, endemia local cujo estudo o levou a conhecer os fatores sociais, econômicos e culturais que favoreciam o seu desenvolvimento. Podemos afirmar que essa perspectiva foi seu grande marco como homem de ciência, que perpassou todas as instituições em que ele teve participação ao longo de cerca de sessenta anos de ininterrupta atuação.

Seu interesse pela esquistossomose deu-se com a criação do Centro de Pesquisas Aggeu Magalhães no Recife, em 1950, instituto de pesquisas aplicadas concebido por um grupo de sanitaristas sob a liderança de Amilcar Barca Pellon e direcionado ao estudo das endemias regionais. Frederico Simões Barbosa participou intensa- mente desse projeto institucional que dirigiu por dois longos períodos (1950-1961 e 1964-1969) e com o qual esteve sempre relacionado, ajudando a torná-lo referência no estudo da esquistossomose e de outras endemias. Ainda nesse período, inseriu seu nome no campo dos estudos entomológicos, quando descreveu novas espécies de Culicoides (maruins) neotropicais, como C. paucienfuscatus, C. alahialinus e C. leoni.

Suas pesquisas sobre epidemiologia da esquistossomose, condições de saúde e produtividade dos cortadores de cana-de-açúcar em Catende (Pernambuco) foram inovadoras. Para o sanitarista, foi o primeiro estudo clínico-epidemiológico realizado no campo, em um contexto onde predominavam os estudos realizados em hospitais e limitados "a apresentação de tabelas casuísticas segundo as principais formas clínicas da doença" 1 (p. 151). As estratégias combinadas de pesquisas da ecologia/biologia dos moluscos e epidemiologia e controle da esquistossomose em populações humanas de regiões do Estado 
de Pernambuco foram bem-sucedidas, e projetaram o trabalho de Frederico Simões Barbosa e sua equipe, que contava com epidemiologistas, técnicos de laboratório e assistentes sociais.

Entre as décadas de 1950 e 1970, como reflexo das atividades desenvolvidas no interior da comunidade científico-acadêmica nacional, bem como nas sociedades especializadas de que fez parte, construiu, concomitantemente, uma significativa carreira de consultor, perito e $m e$ dical officer junto a organismos internacionais que nasceram no pós-Segunda Guerra Mundial, como a Organização Mundial da Saúde (OMS), a Organização Pan-Americana da Saúde (OPAS) e a Organização das Nações Unidas para a Alimentação e a Agricultura (FAO).

No entanto, devemos salientar que, desde a década de 1940, o sanitarista manteve intercâmbios acadêmicos internacionais, quando realizou o mestrado em saúde pública (1946) na Faculdade de Higiene e Saúde Pública da Universidade de Johns Hopkins (Estados Unidos) e estágios em entomologia geral e limnologia na Estação Biológica da Universidade de Michigan e na Divisão de Diptera do Museu Nacional em Washington DC, ambos também nos Estados Unidos.

Da passagem pela OMS, cabe destacar seu papel contestador enquanto parasitologista responsável pela avaliação do uso de moluscicidas no combate à esquistossomose em regiões pobres do planeta, como o continente africano. Lá, participou de uma pesquisa em Gana, refutando relatórios anteriores da instituição que aprovavam o uso do produto no mais importante lago do país. Como ele mesmo relata, foi preciso contrariar os interesses da indústria farmacêutica ao atestar a ineficácia de um método caro e arriscado de controle da doença: "Enfrentei um problema muito grave, porque nas minhas consultorias não indicava os moluscicidas. (...) Os países todos já estavam com alguma consciência voltada para os malefícios produzidos pelos moluscicidas" 3 (p. 301).

Frederico Simões Barbosa também foi coordenador do programa internacional Brasil, Egito e Hungria de pesquisa sobre recursos humanos e atenção primária de saúde, entre 1972 e 1975, após ter retornado ao Brasil, quando, então, iniciou sua trajetória na Universidade de Brasília (UnB), onde se envolveu mais diretamente com essas áreas de investigação.

Na Faculdade de Ciências da Saúde da UnB, como professor de medicina comunitária (19721981), Frederico Simões Barbosa desenvolveu um programa de integração docente assistencial junto a comunidades carentes, trabalho pioneiro que contribuiu para a área de formação de recursos humanos em saúde, combinando conceitos
Foto 2

Frederico Simões Barbosa e funcionários do Centro de Pesquisas Aggeu Magalhães durante visita do Professor Giorgio Schreiber, da Universidade de Minas Gerais, à instituição. Recife, Pernambuco, junho de 1955 (Acervo Casa de Oswaldo Cruz/Fiocruz).

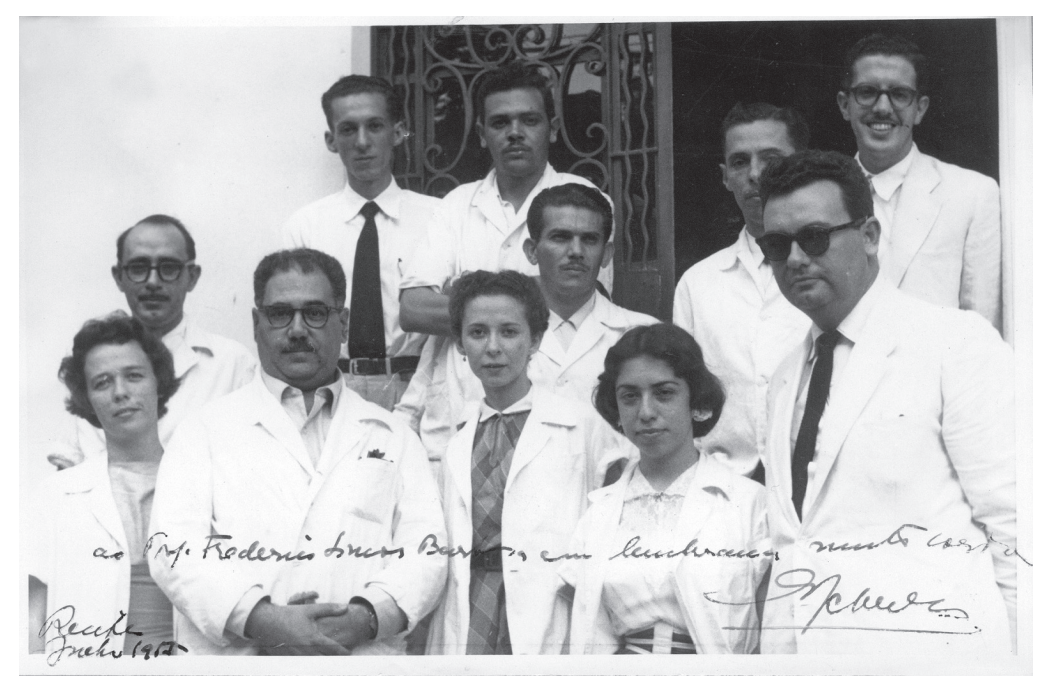

das ciências sociais e das ciências médicas. Segundo ele: "Procurou-se, durante o curso, fazer com que os residentes compreendessem os problemas sociais e de saúde da comunidade, procurando desenvolver nos alunos pensamento crítico sobre os determinantes maiores da doença e as relações entre o componente político/social e a estrutura da prestação de serviços" 4 (p. 32).

Com base nesse paradigma, não foi por acaso a sua importância na fundação da Associação Brasileira de Pós-graduação em Saúde Coletiva (Abrasco), em 1979, entidade comprometida com um novo conceito de saúde pública. Na passagem pela Universidade Federal de São Carlos (UFSCar) e pela Secretaria de Ensino Superior do Ministério da Educação e Cultura (1980-1984), deu continuidade aos estudos realizados na UnB.

Em 1983, ingressou na Escola Nacional de Saúde Pública (ENSP) como professor de epidemiologia e participou das transformações da Fundação Oswaldo Cruz (Fiocruz), conduzidas a partir de 1985 sob a presidência do sanitarista Sérgio Arouca. Foi diretor da escola entre 1985 e 1989 e desempenhou papel central na criação de Cadernos de Saúde Pública (1985), ao lado de Luiz Fernando Ferreira, e do Núcleo de Doenças Endêmicas Samuel Pessoa, depois transformado em departamento (1991), com o objetivo de ser um espaço interdisciplinar dirigido aos estudos 
Foto 3

Frederico Simões Barbosa em trabalho de campo para pesquisa sobre esquistossomose. Pernambuco, década de 1950 (Acervo Casa de Oswaldo Cruz/Fiocruz).

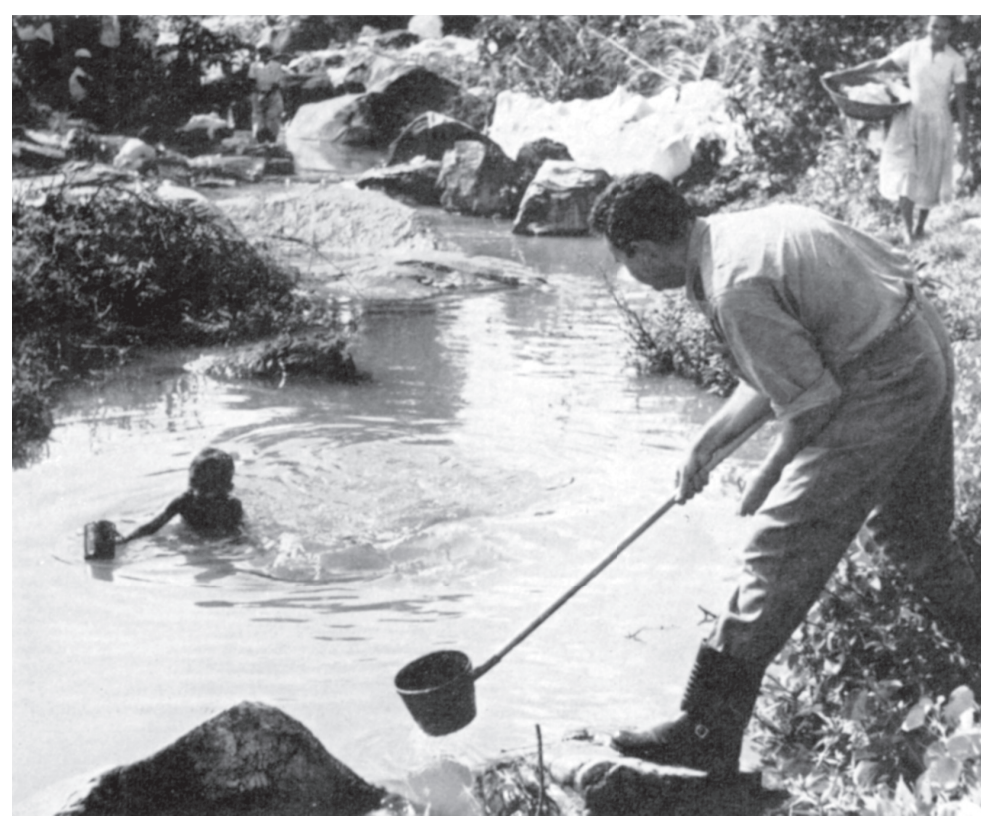

Foto 4

Reunião durante a 4ạ Conferência Nacional de Saúde, realizada na Fundação Ensino Especializado de Saúde Pública, atua ENSP/Fiocruz, de 30 de agosto a 4 de setembro de 1967, no Rio de Janeiro (Acervo Casa de Oswaldo Cruz/Fiocruz). Da esquerda para a direita, Luiz Fernando Ferreira (2의), Frederico Simões Barbosa (3으) e Ernani Braga (6으).

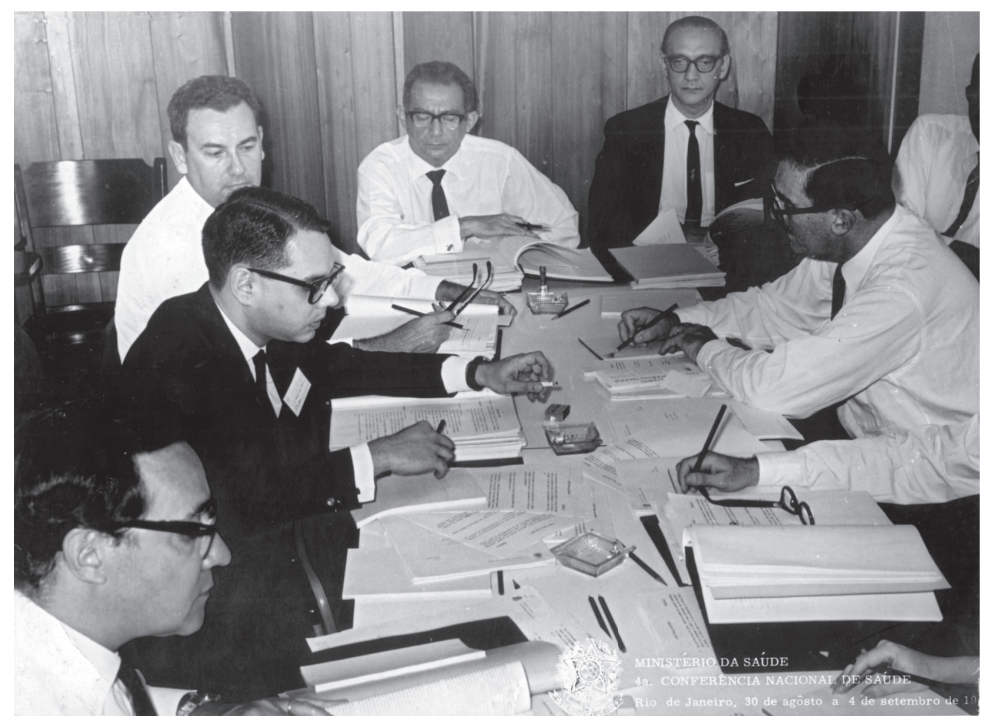


dos determinantes e das estratégias de controle social do processo saúde-doença, em sua dimensão coletiva.

Após a sua aposentadoria em 1991, retornou à casa que ajudou a fundar, o Centro de Pesquisas Aggeu Magalhães (CPqAM), dando continuidade aos estudos que o acompanharam ao longo de sua carreira: epidemiologia e estratégias de controle da esquistossomose.

Ainda no Brasil, foi chefe do Laboratório Clínico do Hospital Militar do Recife (1942); chefe da Divisão Médica do Instituto de Aposentadoria e Pensões dos Empregados em Transportes e Cargas (1945), diretor-geral do Departamento de Saúde Pública de Pernambuco (1945); chefe do Departamento de Medicina Preventiva e da Comunidade - posteriormente transformado em Centro Regional de Investigação e Ensino em Higiene e Saúde Pública (1966-1969); presidente da Sociedade de Medicina de Pernambuco (19671968); diretor da Faculdade de Ciências da Saúde da UnB (1975-1976); presidente da Sociedade Brasileira de Medicina Tropical (1977-1979); consultor de medicina preventiva da Comissão Nacional de Residência Médica do MEC (19791982); professor de medicina preventiva e assessor da Reitoria da UFSCar (1982); editor de CSP (1985-1989); vice-presidente e presidente da Associação Brasileira de Educação Médica - ABEM (1986-1988/1988-1990).

Frederico Simões Barbosa faleceu no Recife em 8 de março de 2004.

\section{Nota}

Este texto resulta dos trabalhos de pesquisa e organização do arquivo pessoal de Frederico Simões Barbosa, realizados no âmbito do projeto História dos 25 Anos da Associação Brasileira de Pós-graduação em Saúde Coletiva (Abrasco), desenvolvido pela Casa de Oswaldo Cruz/Fiocruz, entre os anos de 2004 e 2006, sob a coordenação das pesquisadoras Nísia Trindade Lima e Cristina M. Oliveira Fonseca. Ele foi publicado na revista Patrimônio e Memória (Santos PRE, Mesquita CC, Cruz BDOS, Menezes AP, Reis NRB, Lourenço FS. Saúde pública e ações comunitárias no Arquivo Frederico Simões Barbosa. Patrimônio e Memória 2007; 3:160-70), e está sendo retomado, dez anos depois, com modificações, de forma a ampliar o olhar sobre a trajetória profissional do sanitarista e excluir a análise dos procedimentos metodológicos de organização do seu arquivo pessoal. Ver também: Departamento de Arquivo e Documentação, Casa de Oswaldo Cruz, Fundação Oswaldo Cruz ${ }^{5}$.

\section{Foto 5}

Atividade do Programa Integrado de Saúde Comunitária, coordenado por Frederico Simões Barbosa na Universidade de Brasília. Planaltina (Distrito Federal), 1976-1977 (Acervo Casa de Oswaldo Cruz/Fiocruz).

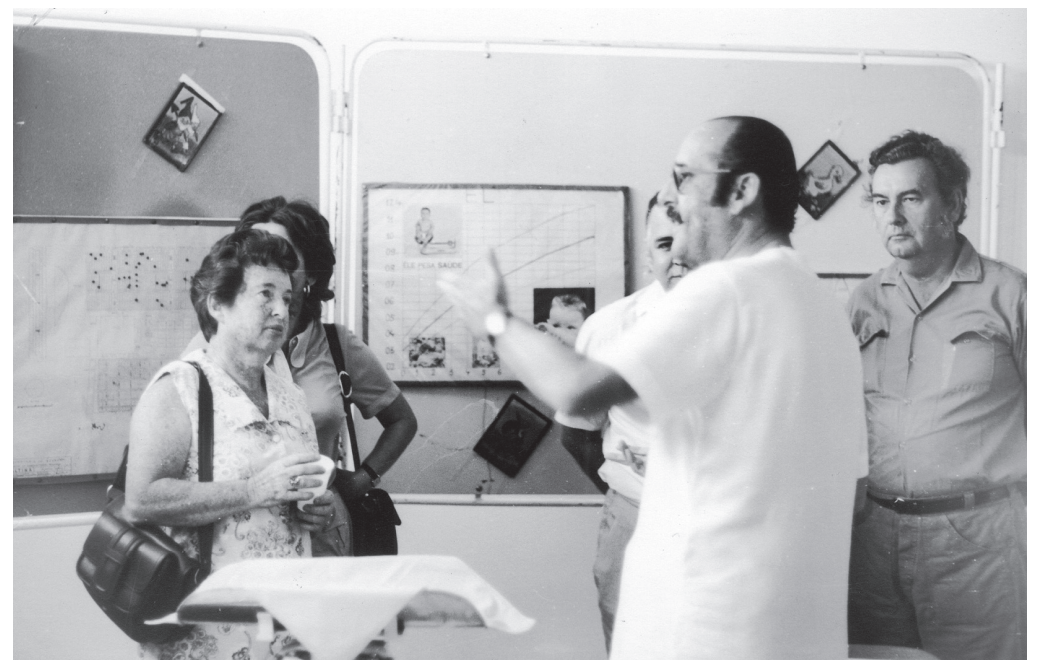

Foto 6

Frederico Simões Barbosa, diretor da ENSP/Fiocruz, e Szachna Cynamon (4o a partir da esquerda), professor da ENSP, durante inauguração do Curso de Saneamento na Vila do João, Rio de Janeiro, março de 1987 (Acervo Casa de Oswaldo Cruz/Fiocruz).

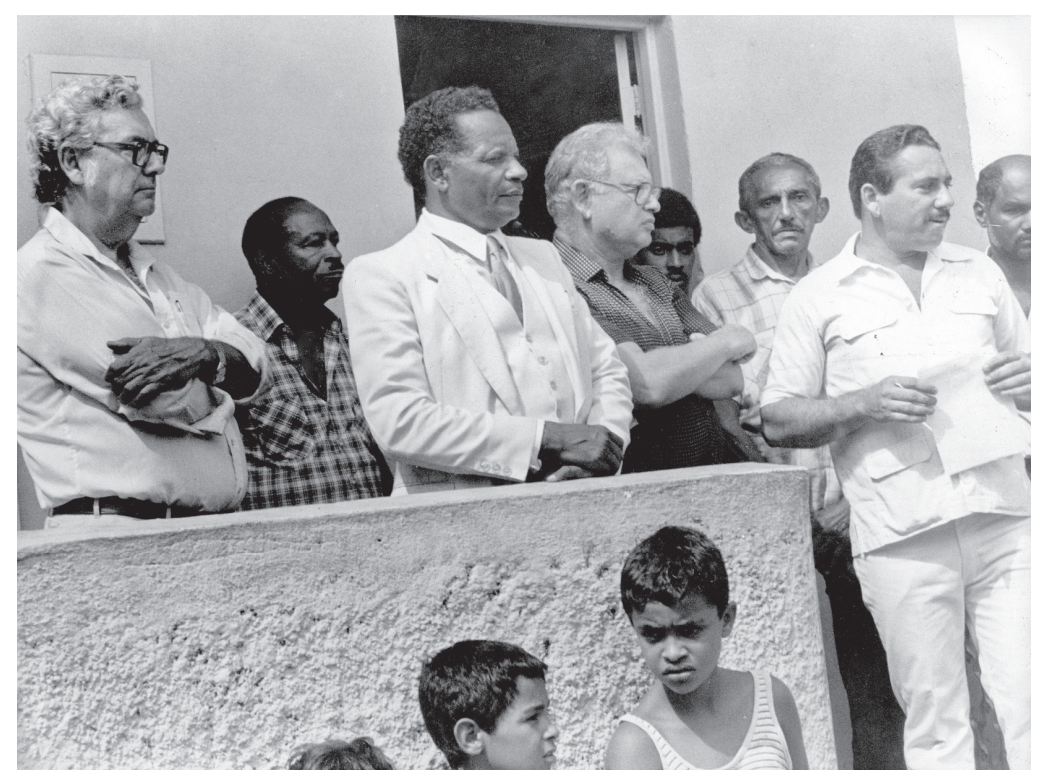




\section{Colaboradores}

Todos os autores participaram igualmente da produção do texto.

1. Coimbra Jr. CEA. Uma conversa com Frederico Simões Barbosa. Cad Saúde Pública 1997; 13:145-55.

2. Paiva CHA. Samuel Pessoa: uma trajetória científica no contexto do sanitarismo campanhista e desenvolvimentista no Brasil. Hist Ciênc Saúde -Manguinhos 2006; 13:795-831.

3. Montenegro AT, Fernandes T, organizadores. Memórias revisitadas: o Instituto Aggeu Magalhães na vida de seus personagens. Rio de Janeiro: Casa de Oswaldo Cruz, Fundação Oswaldo Cruz/Recife: Centro de Pesquisas Aggeu Magalhães, Fundação Oswaldo Cruz; 1997.
4. Barbosa FS. Programa Integrado de Saúde Comunitária: uma história de caso. Brasília: Conselho Nacional de Desenvolvimento Científico e Tecnológico; 1980.

5. Departamento de Arquivo e Documentação, Casa de Oswaldo Cruz, Fundação Oswaldo Cruz. Fundo Frederico Simões Barbosa: inventário. Rio de Janeiro: Fundação Oswaldo Cruz; 2007. (Instrumentos de Pesquisa, 4). 